\title{
OBSERVATORIO
}

\section{MADUREZ DE LA GESTIÓN DE CONTENIDOS, ¿SINÓNIMO DE DESGASTE O DE OPORTUNIDADES?}

\section{Ricardo Eíto-Brun}

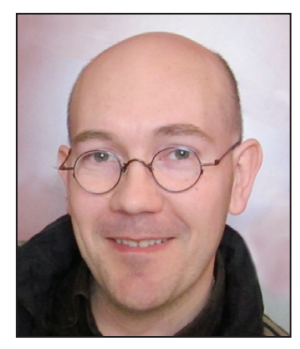

Ricardo Eíto-Brun es doctor en documentación por la Universidad de Zaragoza y licenciado en la misma disciplina por la Universidad de Granada. Desempeña su actividad profesional desde 1996 en el sector de las tecnologías de la información, lo que ha compaginado con la docencia universitaria en la Universidad Carlos III de Madrid. Es autor de varias monografías sobre lenguajes de marcas y gestión de contenidos, y de artículos y contribuciones a congresos y reuniones profesionales. http://orcid.org/0000-0003-1219-0510

Grupo GMV. Departamento de Calidad Isaac Newton 11, P.T.M. 28760 Tres Cantos, Madrid, España reito@gmv.com

\section{Resumen}

Las soluciones técnicas para gestionar contenidos han alcanzado un alto nivel de madurez. Bajo la denominación ECM (enterprise content management) se han agrupado varias aproximaciones técnicas para la gestión integral de la información no estructurada. La aceptación de la gestión de contenidos (GC) en distintos contextos es indicador del valor que estas prácticas aportan a las organizaciones. Pero cabe el riesgo de interpretar esta madurez como sinónimo de desgaste: se debe considerar si todavía hay oportunidades de mejora y nichos de actividad en los que centrar esfuerzos, o si por el contrario queda poco espacio para la innovación. El autor sostiene que una vez se han sentado las bases para el despliegue de este tipo de soluciones y la gestión de contenidos se ha convertido en un término familiar para los usuarios de las tecnologías de la información (TI), existe aún un gran número de oportunidades relacionadas con: a) el análisis del consumo de datos y la experiencia de los usuarios, incluyendo temas complejos como la minería de opiniones, y b) el diseño de soluciones GC que combinen las buenas prácticas de las TI y del área de la gestión de información.

\section{Palabras clave}

Gestión de contenidos, Tecnologías de la información, Gestión de la información, Profesionales de la información.

\section{Title: Content management maturity as a synonym for obsolescence or opportunity?}

\begin{abstract}
Technical solutions for content management have reached a high level of maturity. Under the heading of ECM (enterprise content management), several technical approaches for the management of unstructured information have been grouped. The acceptance of content management in different contexts is indicative of the value that these practices contribute to organizations. There is, however, a risk of interpreting this maturity as a synonym for being past its prime: we should consider whether there are still some opportunities for improvement and niches of activity on which to focus efforts, or if instead there is little room for innovation. The author contends that once we have laid the foundation for the deployment of such solutions and content management has become a familiar term for information technology (IT) users, there remain many opportunities related to: a) the analysis of data usage and user experience, including complex issues such as opinion mining, and $b$ ) the design of ECM solutions that combine best practices in the areas of IT and information management.
\end{abstract}

\section{Keywords}

Content management, Information technologies, Information management, Information professionals.

Eíto-Brun, Ricardo (2013). “Madurez de la gestión de contenidos, ¿isinónimo de desgaste o de oportunidades?”. El profesional de la información, septiembre-octubre, v. 22, n. 5, pp. 377-380.

http://dx.doi.org/10.3145/epi.2013.sep.01 


\section{Mayor alcance de la gestión de contenidos}

Una de las preguntas más frecuentes al revisar los informes de los analistas y estudiar las nuevas características de las aplicaciones informáticas para la gestión de contenidos es si ésta ha alcanzado realmente su madurez. Atendiendo únicamente a los movimientos del mercado, se aprecia dicha madurez, aunque siempre haya incógnitas en torno a las estrategias comerciales de algunas compañías (Wood, 2013). Desde una perspectiva más amplia, se puede apuntar que la madurez alcanzada en aspectos técnicos y la incorporación de la gestión de contenidos en el portfolio de tecnologías de la información de las organizaciones -aún con un alcance limitado- es la cadena de transmisión idónea para identificar nuevas oportunidades profesionales y dotar de un mayor dinamismo a las disciplinas enfocadas en la gestión de la información.

La gestión de contenidos ha evolucionado hacia la integración progresiva de las tecnologías para gestionar información y datos no estructurados.

Inicialmente el término tuvo un significado acotado a un tipo muy concreto de programas informáticos enfocados a la edición colaborativa de sitios web y a la coordinación de los actores que participaban en su diseño y desarrollo.

Posteriormente pasó a englobar herramientas, métodos y procesos de análisis característicos de la gestión documental tradicional. Esto dio al término un significado mucho más amplio. La incorporación de métodos para el análisis de flujos de trabajo, tipos de contenidos, su organización lógica, etc., hizo que la gestión de contenidos se comenzase a entender como una disciplina soportada por soluciones técnicas, y dejó de estar limitada al despliegue de aplicaciones informáticas puntuales. Esta progresión la dotó de un mayor contenido y de una mayor complejidad, y obligó a pensar en la forma de sincronizar y reconciliar la gestión de la información no estructurada con su difusión y publicación a través de diferentes canales, incluyendo los sitios web que han caracterizado la web social.

\section{El foco de la gestión de contenidos ha pasado a ser el repositorio}

Si inicialmente el principal elemento en el que se centraba la atención en cualquier estrategia de gestión de contenidos era el sitio web corporativo, en la actualidad los sitios web son canales a través de los cuales se puede difundir y dar visibilidad de forma selectiva a la información que las organizaciones generan y recopilan. El foco de la gestión de contenidos ha pasado a ser el repositorio: que no está limitado a aquellos datos a los que se ha pensado dar visibilidad a través de internet, sino que engloba toda la información que genera y recibe la organización y es susceptible de ser utilizada -y reutilizada- en la ejecución de procesos de trabajo y en la prestación de servicios a usuarios y clientes.

Este cambio de filosofía se refleja fielmente en los acrónimos que se utilizan al hablar de gestión de contenidos. Se ha establecido una diferencia entre WCM (web content management) y ECM (enterprise content management). EI primero para hacer referencia a la gestión de los contenidos para ser difundidos en la Web, y el segundo como un súper-conjunto que englobaría a la WCM junto a la gestión documental tradicional en sus múltiples variantes: gestión de imágenes digitalizadas o imaging, gestión de registros o records management, gestión de informes y listados generados desde aplicaciones de gestión, etc.

Desde el punto de vista del profesional de la información, esta evolución añade una mayor complejidad a nuestras actividades y obliga a reformular la función y competencias que se precisan para desempeñarlas en este nuevo contexto.

La evolución de la gestión de contenidos añade una mayor complejidad a las actividades del profesional de la información y obliga a reformular su función y competencias

La gestión de contenidos no se ocupa únicamente de la edición de páginas usando plantillas y de la coordinación de equipos de editores, diseñadores gráficos y programadores conocedores de ciertas aplicaciones software. Su alcance se ha ampliado de forma significativa. Un gestor de contenidos debe ser capaz de contextualizar las actividades de la organización, entender y analizar de qué procesos se nutre el repositorio, y qué datos e información se deben exponer y facilitar a los usuarios internos, externos, y a las aplicaciones (incluyendo sitios web).

De todo esto se puede deducir que esta evolución ha tenido como consecuencia:

- un alcance más amplio para la gestión de contenidos, que ha pasado a convertirse en una disciplina de gestión global de información;

- una mayor independencia de la gestión de contenidos respecto a aplicaciones informáticas particulares. Ya no se trata de gestionar procesos de edición y publicación en la Web, sino de gestionar un repositorio que interactúe, mediante protocolos estándares, con múltiples aplicaciones que producen y consumen contenidos;

- la disociación entre gestión de contenidos e información no estructurada. Resulta difícil mantener la validez de esta asociación tradicional;

- la necesidad, desde un punto de vista profesional y académico, de formalizar métodos que permitan analizar y documentar las necesidades de los usuarios y desplegar soluciones para gestionar contenidos basadas en tecnologías.

Otro aspecto que requiere la atención de la comunidad profesional es el diseño de métodos para evaluar la madurez de las prácticas de gestión de contenidos de las organizaciones. Las posibilidades reales de aplicar y poner en práctica esta aproximación generalista se pueden ver afectadas por las necesidades más o menos inmediatas de solventar problemas particulares. Es complejo que una organización considere la gestión de contenidos como una gestión global de sus activos de información. Se están dando pasos en esta línea, y la gestión de contenidos web ha pasado a englobar actividades como el comercio-e, el marketing digital multi- 
canal y la gestión de la experiencia de los usuarios (Myers, 2013; MacComascaigh et al., 2013).

A pesar de ello, es frecuente encontrar bajo el epígrafe "gestión de contenidos" peticiones orientadas al diseño y mantenimiento de espacios web más o menos complejos. Es precisamente aquí donde la creciente complejidad del ecosistema web de las organizaciones ofrece nuevas oportunidades para los profesionales de la información: las relacionadas con el análisis de datos procedentes de las distintas presencias de la organización en internet. En este abanico se incluirían cuestiones tan variadas como el análisis de las visitas, de los patrones de navegación de los usuarios, la minería de datos (textuales y estructurados), o la minería de opiniones para extraer conocimiento de sitios web sociales.

La gestión de contenidos ha pasado a convertirse en una disciplina de gestión global de información

\section{¿Cuál es el límite de la gestión de contenidos?}

La respuesta a esta pregunta sería tan sencilla como decir que depende de qué se entienda por gestión de contenidos. Esta respuesta trivial debería dar lugar a un serio debate entre los profesionales y los académicos que de una u otra forma están involucrados en la gestión de la información y en la formación de futuros profesionales. Obviamente, cuanto más amplia sea la perspectiva desde la que se observe el problema, más completos serán los planes que se tracen para afrontar el futuro. Una visión a corto plazo sólo puede conducir a restricciones y obstáculos que serán difíciles de superar, especialmente para los futuros profesionales en proceso de formación.

Si se limita la gestión de contenidos al diseño y mantenimiento de sitios web y a las actividades de edición de contenidos, se podría afirmar que se ha alcanzado la madurez, tanto desde el punto de vista tecnológico como desde el de las competencias profesionales. La gestión de contenidos sería una commodity (mercancía) más accesible para la mayoría de las organizaciones. Se podría afirmar que se trata de algo plenamente asimilado por las organizaciones, sin que se prevea la necesidad de evolucionar: "un problema ya resuelto" (Antion, 2012).

Si se entiende la gestión de contenidos como una estrategia de gestión global en torno a los activos de información de las organizaciones, cualquiera que sea su origen, encontramos más oportunidades y dificultades. Se deben retomar conceptos como "arquitectura de la organización", "comprensión y modelización de procesos" o "protocolos para la interoperabilidad". Las competencias tecnológicas no deben limitarse al conocimiento de una plataforma de gestión de contenidos determinada, sino a las capacidades de interactuar que ofrecen los estándares y protocolos abiertos. La comprensión y modelización de procesos y los servicios deben enfatizar los aspectos de creación y consumo de información.

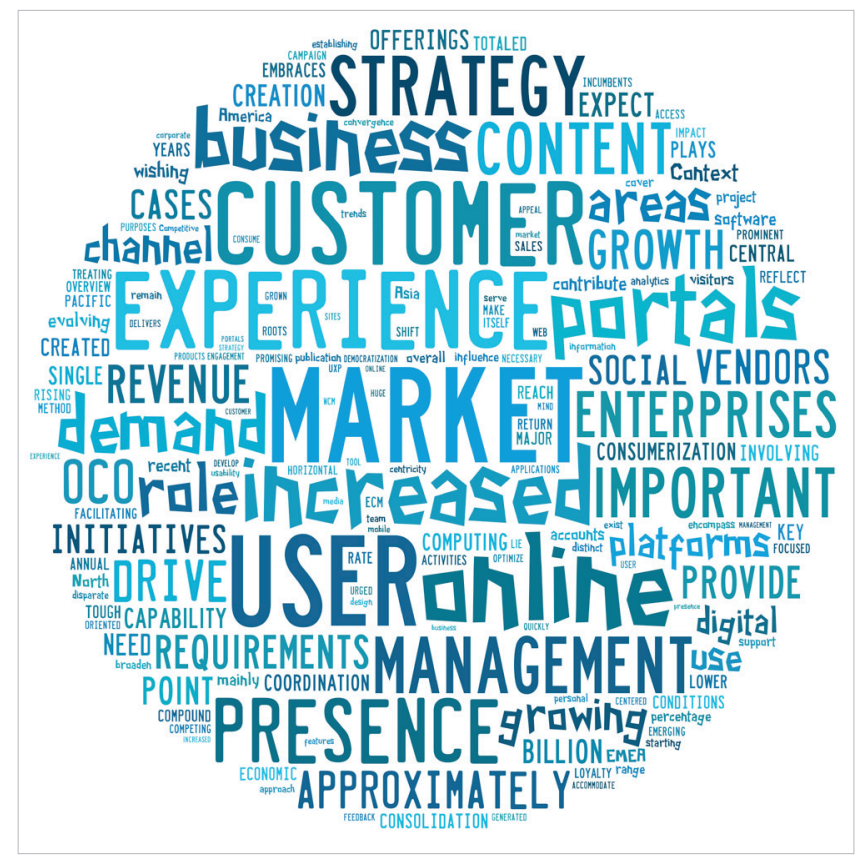

Figura 1. Nube de palabras generada a partir del "cuadrante mágico". Gartner WCM, 31 julio 2013

Si se considera que la gestión de contenidos va más allá de la publicación de contenidos, y que debe incorporar el análisis del uso que se hace de éstos, el mapa de competencias debe incorporar nuevos elementos relacionados con la minería de datos y con la optimización de la presencia de las organizaciones en los distintos canales. La rentabilidad de la presencia en estos canales y la generación de beneficios económicos (más ventas en línea, fidelización de clientes, etc.) se debe incluir en este apartado. En este punto son significativas y recomendables las últimas monografías escritas por Thomas H. Davenport, autor que tuvo años atrás una contribución decisiva en la popularización de la gestión del conocimiento, y que exponen la necesidad de adquirir competencias relacionadas con el análisis cuantitativo de la información o quantitative literacy.

La creciente complejidad del ecosistema web de las organizaciones ofrece nuevas oportunidades para los profesionales de la información

La nube de términos de la figura 1, generada a partir de las conclusiones del "cuadrante mágico" de la consultora Gartner para WCM publicado el 31 de julio de 2013, permite destacar la importancia de conceptos como la experiencia de los usuarios o la presencia en la Web en la estrategia de gestión de contenidos.

Como conclusión, si se mantiene una perspectiva amplia, es complicado afirmar que la gestión de contenidos esté estancada, y podemos resistirnos a verla como una commodity tecnológica más. Ante el amplio número de oportunidades que se presentan, los profesionales de la información deben decidir si están dispuestos a afrontar los retos que plantea el nuevo escenario, y prestar atención a la adquisición de las nuevas competencias que se precisan. 


\section{Bibliografía}

Antion, Daniel (2012). "ECM is dead". AlIM: The global community of information professionals, 19 June.

http://www.aiim.org/community/blogs/expert/ecm-is-dead

Davenport, Thomas H.; Harris, Jeanne G.; Morison, Robert (2010). Analytics at work: smarter decisions, better results. Harvard Business Review Press. ISBN: 9781422177693

Davenport, Thomas H.; Kim, Jinho (2013). Keeping up with the quants: your guide to understanding and using analytics. Harvard Business Review Press. ISBN: 9781422187258

MacComascaigh, Mick; Gilbert, Mark R.; Murphy, Jim; Tay, Gavin (2013). "Magic quadrant for web content manage- ment". Gartner, 31 July.

http://www.gartner.com/technology/reprints.do?id=1$1 / 3$ UYPS\&ct $=130802 \& s t=s b$

Myers, Anthony (2013). "Gartner MQ for web content management 2013: Adobe, SDL, SiteCore Lead + Market overview". CMSWire, 1 August.

http://www.cmswire.com/cms/customer-experience/ gartner-mq-for-web-content-management-2013-adobe-sdlsitecore-lead-market-overview-021950.php

Wood, Rich (2013). "Reports of SharePoint's death are greatly exaggerated". CMSWire, 22 August.

http://www.cmswire.com/cms/social-business/reports-ofsharepoints-death-are-greatly-exaggerated-022206.php

\title{
Anuario ThinkEPI 2013
}

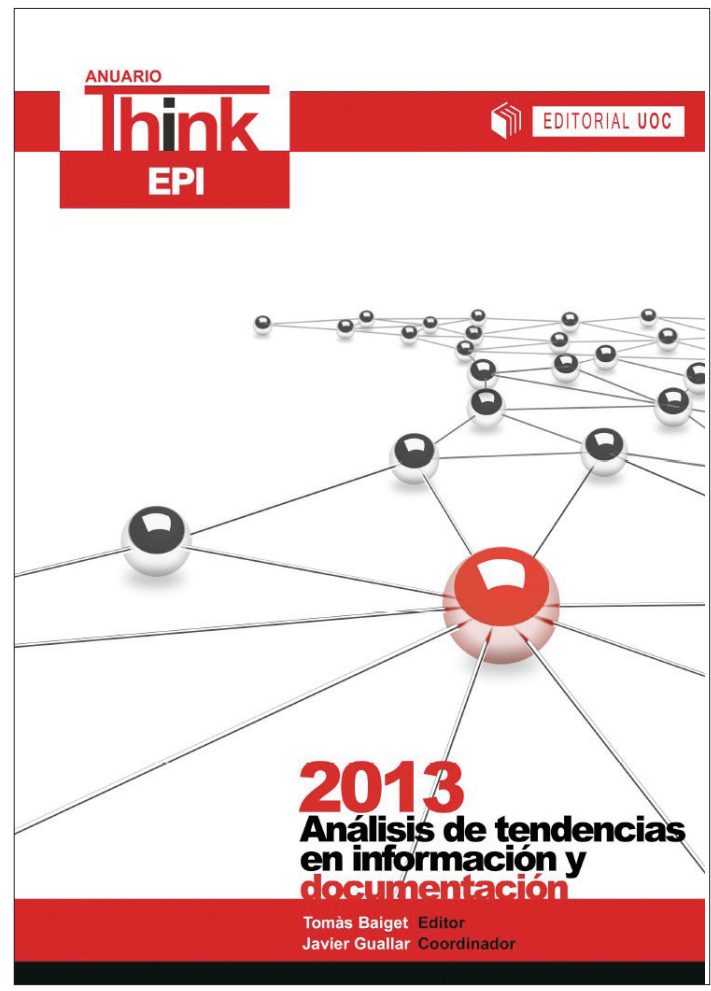

\author{
272 páginas de análisis de \\ tendencias en información, \\ documentación y \\ comunicación
}

Formulario de compra:

http://www.thinkepi.net/solicitud

Información y pedidos:

Isabel Olea

epi.iolea@gmail.com

+34-608 491521 